\title{
Critical Adsorption of Polyelectrolytes onto Charged Spherical Colloids
}

\author{
Roland G. Winkler ${ }^{1}$ and Andrey G. Cherstvy ${ }^{1,2}$ \\ ${ }^{1}$ Institut für Festkörperforschung, Forschungszentrum Jülich, D-52425 Jülich, Germany \\ ${ }^{2}$ Max-Planck-Institut für Physik komplexer Systeme, Nöthnitzer Straße 38, D-01187 Dresden, Germany
}

(Received 8 September 2005; published 13 February 2006)

\begin{abstract}
The adsorption of a flexible polyelectrolyte in a salt solution onto an oppositely charged spherical surface is investigated. An analytical solution is derived, which is valid for any sphere radius and consistently recovers the result of a planar surface in the limit of large sphere radii, by substituting the Debye-Hückel potential via the Hulthén potential. Expressions for critical quantities such as the critical radius and the critical surface charge density are provide. A comparison of our theoretical results with experiments and computer simulations yields remarkable good agreement.
\end{abstract}

The complexation of charged macroions by oppositely charged polyelectrolytes is a fundamental process in biological systems and many technical applications. Particular examples are the complexation of histone proteins by DNA in nucleosomal core particles [1,2] as well as complexes of polyelectrolytes with charged colloids and micelles [3]. Industrial applications are as diverse as stabilization of colloidal suspensions, water treatment, and paper making [4].

The understanding of the complexation between a polyion and a macroion surface accompanied by screening effects due to counterions and salt posses a major theoretical challenge [5]. Despite significant efforts and progresses [5-11], the understanding of charged complexes is still unsatisfactory and lacks behind that of neutral complexes. Certain insight into the complexation process is typically obtained by approximation schemes, e.g., variational calculations [6-9], which, however, may lead to controversial results [12] and often apply only in limiting situations such as pointlike particles [9] or large colloidal radii [6].

The theoretical studies of the adsorption behavior of polyelectrolytes onto spherical surfaces $[7,10,11,13,14]$ lead to the observation of a phase-transition-like behavior; i.e., a bound polymer state appears at certain critical conditions which depend on, e.g., the sphere radius and screening of the Coulomb interaction. The variational calculations of Muthukumar and his co-workers [6,8] have provided useful insight into this transition in the limit of large sphere radii. Experiments on polyelectrolyte-protein and -micelle complexes [15] and computer simulations [14], however, typically yield dependencies of the critical quantities on the Debye screening length which deviate from the theoretical predictions. To understand and interpret the experimental results correctly, an analytical solution of the adsorption problem valid for any sphere radius is mandatory.

In this Letter, we will present an exact solution for the critical adsorption of a flexible polyelectrolyte onto an oppositely charged spherical macroion. Expressions for critical quantities are provided, which are valid for any sphere radius. In particular, in the limit of zero macroion curvature, the results for a planar surface are obtained. In general, we find a significantly different dependence of the critical surface charge density on the Debye screening length than theoretically predicted before, but our predictions are consistent with experimental findings.

The weakly charged polyelectrolyte is described by a continuous space curve with the linear charge density $\rho$. The intramolecular Coulomb and excluded volume interactions are not taken into account explicitly but are rather adsorbed into the Kuhn segment of length $l$. Expressions for its dependence on the Debye screening length are provided in Ref. [6]. The oppositely charged macroion is considered a spherical particle of radius $a$ with a homogeneous surface charge density $\sigma$. The differential equation for the probability density $G\left(\boldsymbol{r}, \boldsymbol{r}^{\prime} ; L\right)$ (Green function) of a flexible polymer of length $L$ with one end point located at $\boldsymbol{r}(0)=\boldsymbol{r}^{\prime}$ and the other end point located at $\boldsymbol{r}(L)=\boldsymbol{r}$ is given by

$$
\left(\frac{\partial}{\partial L}-\frac{l}{6} \Delta_{r}+\frac{V(\boldsymbol{r})}{k_{B} T}\right) G\left(\boldsymbol{r}, \boldsymbol{r}^{\prime} ; L\right)=\delta\left(\boldsymbol{r}-\boldsymbol{r}^{\prime}\right) \delta(L)
$$

with $k_{B}$ the Boltzmann constant and $T$ the temperature [16-18]. $V$ is the interaction energy per length of the chain end with the sphere, which we take as the Debye-Hückel potential

$$
V_{\mathrm{DH}}=-\frac{4 \pi a^{2}|\sigma \rho|}{\epsilon(1+\kappa a)} \frac{e^{-\kappa(r-a)}}{r},
$$

where $r$ is the radial distance from the sphere center and $\kappa$ is the inverse Debye screening length. Equation (1) has to be solved with the boundary conditions $G=0$ at the surface and $\lim _{|r| \rightarrow \infty} G=0$.

To find a solution of Eq. (1) we use the bilinear expansion

$$
G\left(\boldsymbol{r}, \boldsymbol{r}^{\prime} ; L\right)=\sum_{n} \psi_{n}^{*}\left(\boldsymbol{r}^{\prime}\right) \psi_{n}(\boldsymbol{r}) e^{-\lambda_{n} L}
$$

in terms of the eigenfunctions $\psi_{n}$ of the eigenvalue equation 


$$
\left(-\frac{l}{6} \Delta_{r}+\frac{1}{k_{B} T} V_{\mathrm{DH}}(\boldsymbol{r})\right) \psi_{n}(\boldsymbol{r})=\lambda_{n} \psi_{n}(\boldsymbol{r})
$$

with the corresponding eigenvalues $\lambda_{n}$. As is well known, in the limit $L / l \gg 1$ the Green function is dominated by the eigenfunction corresponding to the ground state. Thus, we can restrict our considerations to the lowest eigenvalue [16-18].

Equation (4) corresponds to the Schrödinger equation of a particle in a spherically symmetric potential. Hence, the ground state eigenfunction ( $s$ wave) is a function of the radial coordinate only $\left[\psi_{0}(\boldsymbol{r})=\psi_{0}(r)\right]$. But even for the ground state, there is no analytical solution for $G$.

To obtain an analytical solution, we approximate the Debye-Hückel potential by the Hulthén potential $[19,20]$ in the following way:

$$
\frac{e^{-\kappa(r-a)}}{r} \rightarrow \frac{e^{-\kappa(r-a)}\left(1-e^{-\kappa a}\right)}{a\left(1-e^{-\kappa r}\right)}
$$

i.e., the potentials are identical for $r=a$. Moreover, the difference between the two expressions is small for $\kappa a \leq$ $\kappa r \ll 1$ as well as for $\kappa a \gg 1$, since in the latter limit $r$ in the denominator is slowly varying and can be replaced by $a$ and $e^{-\kappa r} \ll 1$.

With the substitutions $\psi_{0}(r)=e^{-\xi_{0} \kappa r}\left(1-e^{-\kappa r}\right) \chi(r) / r$ and $x=1-e^{-\kappa r}$, Eq. (4) turns into the hypergeometric differential equation

$$
\begin{aligned}
x(1-x) \frac{d^{2}}{d x^{2}} \chi(x)+[2- & \left.x\left(3+2 \xi_{0}\right)\right] \frac{d}{d x} \chi(x) \\
- & \left(1+2 \xi_{0}-\frac{2}{p}\right) \chi(x)=0,
\end{aligned}
$$

for $\chi$, where the eigenvalue $\lambda_{0}$ is related to $\xi_{0}$ via $\lambda_{0}=$ $-l \xi_{0}^{2} \kappa^{2} / 6$ and $p=\kappa^{2} \epsilon k_{B} T l(1+\kappa a) /\left[12 \pi a|\sigma \rho|\left(e^{\kappa a}-\right.\right.$ 1)]. The solution of Eq. (6) around the point $x=1$ is given by $\chi(x)=F(\alpha, \beta ; \alpha+\beta-\gamma+1 ; 1-x)$, with $F$ the Gauss hypergeometric function and the abbreviations $\alpha=$ $\xi_{0}+1-\sqrt{\xi_{0}^{2}+2 / p}, \quad \beta=\xi_{0}+1+\sqrt{\xi_{0}^{2}+2 / p}, \quad$ and $\gamma=2[21]$.

The eigenvalue $\lambda_{0}$ (or $\xi_{0}$ ) is determined from the boundary conditions. In general, the eigenvalues of Eq. (4) can be positive and negative [16]. The positive eigenvalues correspond to free states $\left(\xi_{0} \in \mathbb{C}, \psi_{0}\right.$ is a periodic function) and negative ones to bound states $\left(\xi_{0} \in \mathbf{R}, \psi_{0}\right.$ decays for $r \rightarrow$ $\infty)$. The transition between free and bound states appears for $\lambda_{0}=\xi_{0}=0$. Since $F(\alpha, \beta ; \alpha+\beta-\gamma+1 ; 1-x)=$ 0 converges for $|1-x|<1$, and $\alpha+\beta-\gamma+1$ is neither zero nor a negative integer, the boundary condition for $r \rightarrow$ $\infty$ (i.e., $x=1$ ) is satisfied. Hence, the eigenvalue $\xi_{0}$ is determined by the boundary condition $F(\alpha, \beta ; \alpha+\beta-$ $\left.\gamma+1 ; e^{-\kappa a}\right)=0$. For $\xi_{0}=0$, the dimensionless parameter $p$ then assumes a particular value $p_{c}$ for a given $\kappa a$, which is determined from the condition

$$
F\left(1-\sqrt{2 / p_{c}}, 1+\sqrt{2 / p_{c}} ; 1 ; e^{-\kappa a}\right)=0 .
$$

The numerical solution, presented in Fig. 1, displays a monotonic decrease of $p_{c}$ with increasing $\kappa a$. For small $\kappa a$, the critical values are well approximated by $p_{c} \approx 2-$ $4 \kappa a$. This dependence is consistent with the necessary condition for the existence of zeros for $F$, namely, $p<2$ [22].

In the limit $\kappa a \rightarrow \infty$, the boundary condition (7) can be expressed in terms of Legendre functions of the first kind $P_{\nu}$ and the Bessel function of the first kind $J_{0}$ according to $\lim _{\kappa a \rightarrow \infty} F\left(\alpha_{c}, 2-\alpha_{c} ; 1 ; e^{-\kappa a}\right)=\lim _{\kappa a \rightarrow \infty} P_{-\alpha_{c}}(1-$ $\left.2 e^{-\kappa a}\right)=\lim _{\kappa a \rightarrow \infty} J_{0}\left(\sqrt{8 / p_{c}} e^{-\kappa a / 2}\right)=0$, with $\alpha_{c}=1-$ $\sqrt{2 / p_{c}}$ [21]. The latter condition is identical to the boundary condition for the adsorption of a polyelectrolyte onto a planar surface [16]. Thus, our approach reproduces exactly the properties of the planar geometry in the limit of vanishing sphere curvature. Denoting the first positive root of $J_{0}$ by $j_{0}$, where $j_{0}=2.4048 \ldots$, we obtain the approximation $p_{c}=\left(8 / j_{0}^{2}\right) e^{-\kappa a}$ for large $\kappa a$.

Using the critical values $p_{c}$, we can calculated other critical quantities for the adsorption, such as the critical temperature [6], the critical colloid surface charge density $\left(\sigma_{c}\right)$, or the critical colloid radius $a_{c}$. As pointed out in Ref. [23], the critical surface charge density is the more useful experimental quantity than the critical temperature, because a temperature change affects not only the colloid polymer interaction but also the solvent properties.

Figure 2 displays the critical charge density as a function of $\kappa$. For $|\sigma|>\left|\sigma_{c}\right|$, polyelectrolytes adsorb at the spherical macroion. In the large curvature limit $(\kappa a \ll 1)$, we obtain the critical charge density

$$
\left|\sigma_{c}\right|=\frac{\epsilon k_{B} T l}{24 \pi a^{2}|\rho|} \kappa
$$

when we use $p_{c}=2$. Thus, the exact solution for the

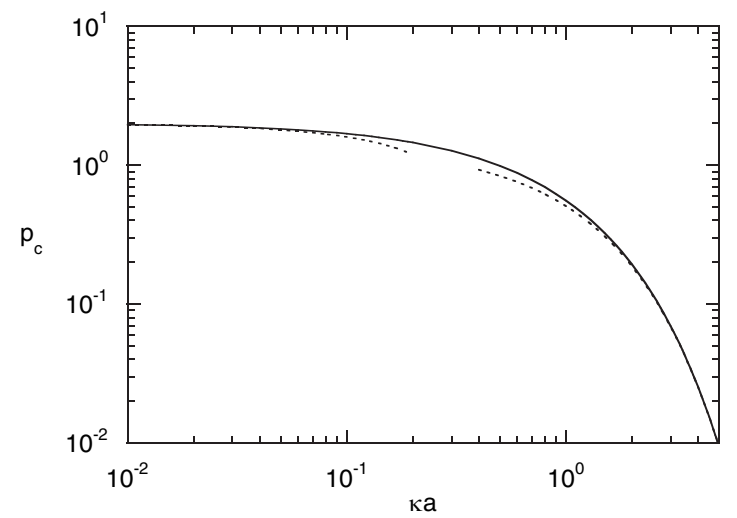

FIG. 1. Critical values $p_{c}$ obtained from the boundary condition at the sphere surface. The dotted lines are the approximations $p_{c} \approx 2-4 \kappa a$ for $\kappa a \ll 1$ and $p_{c}=\left(8 / j_{0}^{2}\right) e^{-\kappa a}$, where $j_{0}=2.4048 \ldots$, for $\kappa a \gg 1$, respectively. 


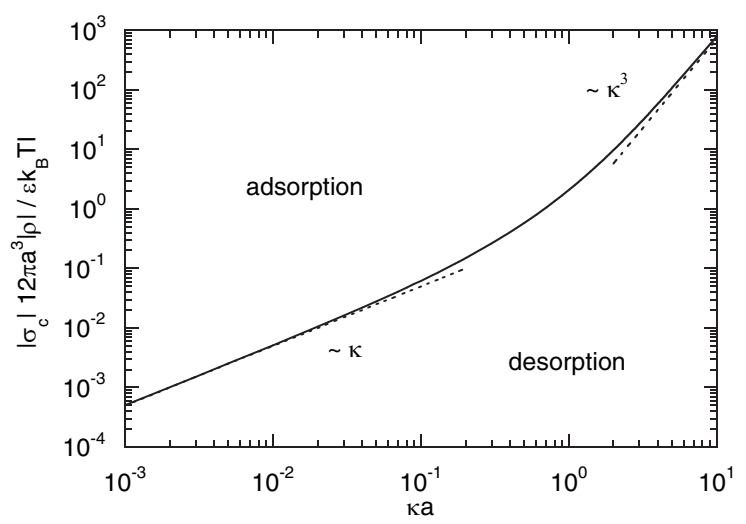

FIG. 2. Critical charge $\left|\sigma_{c}\right|$ as function of the inverse Debye screening length. The dotted lines are the analytical approximations Eq. (8) for $\kappa a \ll 1$ and Eq. (9) for $\kappa a \gg 1$, respectively. No adsorption is obtained in the area to the right of the curve.

Debye-Hückel potential predicts a linear dependence of the critical colloid charge density on the inverse Debye screening length for $\kappa a \ll 1$. This is different from the predicted dependence $\left|\sigma_{c}\right| \sim \kappa^{2}$ based on the variational calculation of Ref. [6]. However, the variational calculation of Ref. [7] predicts the same dependence on $\kappa$, but the numerical factors are rather different. The Kuhn segment length $l$ of the polymer is independent of $\kappa$ in this limit, as shown in Ref. [17].

In the opposite limit of small curvature $(\kappa a \gg 1)$, we obtain from $p_{c}=\left(8 / j_{0}^{2}\right) e^{-\kappa a}$ the critical charge density

$$
\left|\sigma_{c}\right|=\frac{j_{0}^{2} \epsilon k_{B} T l}{96 \pi|\rho|} \kappa^{3}
$$

This dependence on $\kappa$ is identical to that found by variational calculations [6,7]. Taking the dependence of the Kuhn length on $\kappa$ into account - for flexible polymers, $l \sim$ $\kappa^{-b_{l}}$ with $b_{l}=4 / 5-5 / 4$ is predicted $[17,24]$-we find $\left|\sigma_{c}\right| \sim \kappa^{-(11 / 5-7 / 4)}[6]$.

The limit $\kappa a \rightarrow \infty$ corresponds to the limit of a planar surface, and we obtain exactly the same expression as that derived for such a geometry in Refs. [16,17]. However, these authors consider a planar surface with surface charge densities on both sides of the plane. The small curvature limit of our potential corresponds to a charge density on one of the surfaces only. This leads to a $\left|\sigma_{c}\right|$ which is a factor of 2 larger than that presented in Refs. [16,17].

The complex formation of a polyelectrolyte with oppositely charged micelles and proteins has been studied in, e.g., Refs. [15,23,25-27]. These experiments confirm that complexation occurs only when the surface charge density exceeds a critical value. This value typically grows with the reciprocal Debye screening length as $\left|\sigma_{c}\right| \sim \kappa^{b}$ with $b=1-1.4$ [15,25-27]. Our results agree with the experimental findings when we take the above $\kappa$ dependence of $l$ into account. Since $1 \lesssim \kappa a \lesssim 5$ for the experimental systems, their $\sigma_{c}$ 's are rather within the crossover regime than characteristic for the limiting behavior at small or large $\kappa a$. The slope of the curve in Fig. 2 monotonously increases from 2 to 2.8 in this $\kappa a$ interval. A power-law fit for $1 \leq$ $\kappa a \leq 4$ yields $\left|\sigma_{c}\right| \sim \kappa^{1.4}$, when we use $b_{l}=1$, in agreement with experiments.

Instead of the charge density $\sigma_{c}$, a critical sphere radius $a_{c}$ is often considered $[6,8,14]$. By introducing the abbreviation $\bar{\kappa}=\left[96 \pi|\sigma \rho| /\left(j_{0}^{2} \epsilon k_{B} T l\right)\right]^{1 / 3}$ and using the definition of $p$, we obtain the following equation for $a_{c}$ :

$$
p_{c}\left(\kappa a_{c}\right)\left(e^{\kappa a_{c}}-1\right)-\frac{8 \kappa^{2}}{j_{0}^{2} a_{c} \bar{\kappa}^{3}}\left(1+\kappa a_{c}\right)=0 .
$$

The solution of this equation yields a universal curve for $a_{c} \bar{\kappa}$ as a function of $\kappa / \bar{\kappa}$.

The numerical solution of Eq. (10) is shown in Fig. 3 together with the analytical approximations

$$
a_{c} \bar{\kappa}=\left\{\begin{array}{ll}
\sqrt{4 \kappa / \bar{\kappa} j_{0}^{2}} & \kappa \ll \bar{\kappa} \\
(\kappa / \bar{\kappa})^{2} /\left[1-(\kappa / \bar{\kappa})^{3}\right] & \kappa \rightarrow \bar{\kappa}
\end{array} .\right.
$$

No adsorption is obtained in the region located at the right of the curve. At a fixed $\kappa<\bar{\kappa}$, the entropy penalty due to adsorption of the chain monomers decreases with increases sphere radius. Beyond the critical radius the energy gain exceeds the entropy loss and the polymer adsorbs at the sphere surface. As is obvious from the analytical expression, $\bar{\kappa}$ is the maximum value of the inverse Debye screening length; no adsorption is obtained for larger values, neither for a sphere nor for a planar surface. This is qualitatively consistent with the variational calculations of Refs. $[6,8]$. These calculations predict the same dependence of the maximum value for $\kappa$ on the polymer and sphere parameters as our (exact) solution. Quantitatively, however, the value of the variational calculation is smaller

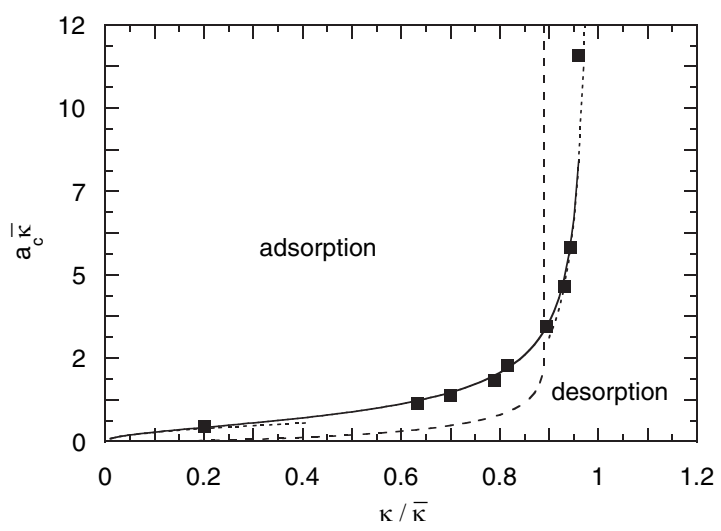

FIG. 3. Critical radius $a_{c}$ as a function of the Debye screening length according to Eq. (10). The dotted lines are the approximations of Eq. (11). The dashed line represents the critical radius according to the variational calculations of Refs. [6,8]. The symbols are Monte Carlo simulation results taken from Ref. [14]. To match the scales of our model, the $\kappa$ values of Ref. [14] are multiplied by 4.06 and the values for the critical radii are divided by 16 . 
by the factor $8 / j_{0}^{2}$. In addition, the shape of the critical curve is rather different as shown in Fig. 3.

In Ref. [14], the critical radius has been determined by Monte Carlo simulations. By adjusting the $\kappa$ values of these data such that the singularity appears at unity, we find that the functional dependence of the simulation data is very similar to that predicted by our calculations. Multiplying the simulation data by a proportionality factor, we achieve a remarkable good agreement with our universal $a_{c}$ curve. Hence, the difference between the simulation data and the results of the variational calculations of the Muthukumar model is not due to the ground state dominance approximation as speculated in Ref. [14]. It is rather a consequence of the limited applicability of the variational ansatz.

An important issue of polyelectrolyte adsorption is overcharging of a spherical object. Several theoretical models predict such an effect [28-30] and it has been observed experimentally in complexation of polyelectrolytes with globular proteins of inhomogeneous charge patterns [27]. Our considerations apply to the adsorption transition only and no overcharging can be predicted because there is a critical charge density below which no adsorption occurs. The overcharging phenomena requires further investigations.

In the present model the regime of weak polyelectrolyte adsorption onto a sphere is considered. In the opposite limit of strong adsorption, the adsorbed polyelectrolytes self-organize in well-defined patterns on the sphere surface [13]. Here, the chain entropy becomes less important; the pattern is rather governed by maximization of polyelectrolyte-sphere attraction and minimization of polyelectrolyte-polyelectrolyte repulsion. Within a simple model, the solution of the linear Poisson-Boltzmann equation for such charge patterns predicts that neutral and undercharged complexes are favored [30,31].

In summary, we have derived critical values for the adsorption of a weakly charged flexible polyelectrolyte onto an oppositely charged spherical surface. In the limit of zero curvature, the results for a planar surface are consistently obtained. The experimentally determined power-law dependencies of the critical charge density on the Debye screening length can be explained when the dependence of the Kuhn length on the screening length is taken into account. The comparison of the analytically predicted universal curve for the critical sphere radius with computer simulations [14] yields remarkable good agreement. More experiments are required to fully understand the adsorption of flexible polymers. We hope that the predicted universal dependencies, e.g., for the critical sphere radius (10), will be helpful in such an endeavor.
[1] B. Alberts, D. Bray, J. Lewis, M. Raff, K. Roberts, and J.D. Watson, Molecular Biology of the Cell (Garland, New York, 1994).

[2] M. V. Volkenstein, Molecular Biophysics (Academic Press, New York, 1977).

[3] Macromolecular Complexes in Chemistry and Biology, edited by P.L. Dubin, J. Bock, R. M. Davis, D. Schulz, and C. T. (Springer Verlag, Berlin, 1994).

[4] D.H. Napper, Polymeric Stabilization of Colloidal Dispersions (Academic Press, New York, 1983).

[5] H. Boroudjerdi, Y.-W. Kim, A. Naji, R. R. Netz, X. Schlagberger, and A. Serr, Phys. Rep. 416, 129 (2005).

[6] F. van Goeler and M. Muthukumar, J. Chem. Phys. 100, 7796 (1994).

[7] P. Haronska, T. A. Vilgis, R. Grottenmüller, and M. Schmidt, Macromol. Theory Simul. 7, 241 (1998).

[8] C. Y. Kong and M. Muthukumar, J. Chem. Phys. 109, 1522 (1998).

[9] E. Gurovitch and P. Sens, Phys. Rev. Lett. 82, 339 (1999).

[10] T. Wallin and P. Linse, J. Phys. Chem. B 101, 5506 (1997).

[11] E. M. Mateescu, C. Jeppesen, and P. Pincus, Europhys. Lett. 46, 493 (1999).

[12] R. Golestanian, Phys. Rev. Lett. 83, 2473 (1999).

[13] K.-K. Kunze and R. R. Netz, Phys. Rev. Lett. 85, 4389 (2000).

[14] P. Chodanowski and S. Stoll, J. Chem. Phys. 115, 4951 (2001).

[15] H. Zhang, K. Ohbu, and P. L. Dubin, Langmuir 16, 9082 (2000).

[16] F. W. Wiegel, J. Phys. A 10, 299 (1977).

[17] M. Muthukumar, J. Chem. Phys. 86, 7230 (1987).

[18] M. Doi and S.F. Edwards, The Theory of Polymer Dynamics (Oxford University, Oxford, 1986).

[19] L. Hulthén, Ark. Mat. Astron. Fys. 28A, No. 5 (1942).

[20] C. S. Lam and Y. P. Varshni, Phys. Rev. A 4, 1875 (1971).

[21] I. S. Gradshteyn and I. M. Ryzhik, Table of Integrals, Series, and Products (Academic Press, San Diego, 1980).

[22] A. Gil, W. Koepf, and J. Segura, Numerical Algorithms 36, 113 (2004).

[23] Y. Li, P.L. Dubin, R. Spindler, and D. A. Tomalia, Macromolecules 28, 8426 (1995).

[24] M. Ullner, J. Phys. Chem. B 107, 8097 (2003).

[25] X.H. Feng, P.L. Dubin, H.W. Zhang, G. F. Kirton, P. Bahadur, and J. Parotte, Macromolecules 34, 6373 (2001).

[26] N. Miura, P.L. Dubin, C. N. Moorefield, and G. R. Newkome, Langmuir 15, 4245 (1999).

[27] K. W. Mattison, P. L. Dubin, and I. J. Brittain, J. Phys. Chem. B 102, 3830 (1998).

[28] R. R. Netz and J.-F. Joanny, Macromolecules 32, 9026 (1999).

[29] T. T. Nguyen and B. I. Shklovskii, J. Chem. Phys. 115, 7298 (2001).

[30] A. G. Cherstvy and R. G. Winkler, J. Phys. Chem. B 109, 2962 (2005).

[31] A. G. Cherstvy and R. G. Winkler, J. Chem. Phys. 120, 9394 (2004). 